\title{
Influence of carbohydrate and fat intakes on high-protein-induced effects on body weight and insulin sensitivity in rats
}

\author{
L. Chevalier, C. Bos, D. Azzout-Marniche, G. Fromentin, D. Tomé and C. Gaudichon \\ UMR914 INRA-AgroParisTech Physiologie de la Nutrition et du Comportement Alimentaire, Paris, France
}

\begin{abstract}
High-protein diets have proved beneficial for controlling weight and improving many metabolic variables ${ }^{(1)}$. However, the macronutrient exchanges necessary to increase dietary protein level are potential confounding factors in these effects. Moreover, results from animal studies are often obtained by using diets with a very high protein content ( $>50 \%$ energy) and are difficult to extrapolate to human subjects. The aim of the present work was to determine the influence of carbohydrate (CHO):fat in the high-protein-diet-induced modulation of food intake, body weight and metabolic variables in rats submitted to normal-protein, moderately-high- (33\% energy) or very-high- (50\% energy) protein diets.

Male Wistar rats $(n$ 104) were adapted to different diets with normal, intermediate and high levels of protein (NP, IP and HP respectively) and different CHO:fat. During the 2 weeks of ad libitum feeding body weight and consumption were monitored every $2 \mathrm{~d}$. At the end of this period blood was sampled in the fasted state to measure glycaemia and hormones (insulin, glucagon, leptin). The gastrocnemius muscle mass was weighed. The HOMA index was calculated as insulin $(\mathrm{pm}) \times$ glycaemia $(\mathrm{mm}) / 22.5$ to assess insulin sensitivity.

Voluntary energy intakes were significantly decreased with increasing protein level, independently of the other macronutrients: IP and HP rats consumed 14 and $22 \%$ less energy than NP rats $(P<0.0001)$. Accordingly, weight gain and leptinaemia were significantly reduced with increasing dietary protein level (weight gain of 70, 60 and $51 \mathrm{~g}$ and leptinaemia of 680, 597 and 419 pM for NP, IP and HP rats respectively), independently of the CHO:fat intake, while the relative weight of gastrocnemius muscle was positively correlated with protein level $(P<0.0001)$, indicating a decrease in adiposity. Glycaemia was neither influenced by the protein level nor by $\mathrm{CHO}$ :fat. Plasma insulin and glucagon levels were influenced by both dietary protein $(P<0.05)$ and CHO:fat $(P<0.02)$. The HOMA index was only significantly influenced by CHO:fat intake $(P<0.005)$.

Increasing protein intake is the primary factor responsible for the consistent reduction observed in energy intake, weight gain, adiposity and leptin levels. The study also shows that the influence of dietary protein on these factors is dose dependent. Conversely, the improvement in insulin sensitivity occurring with high-protein low-CHO diets appears to primarily result from the decreased $\mathrm{CHO}$ content and only indirectly from the increased protein level.
\end{abstract}

Study supported by Arilait Recherches.

1. Lacroix M, Gaudichon C, Martin A, Morens C, Mathé V, Tomé D \& Huneau JF (2004) Am J Physiol Regul Integr Comp Physiol 287, R934-R942. 